\title{
Silicon nanowires (SiNWs) surface engineering potential for bioenergy
}

\begin{abstract}
To date, many efforts have been made to build bioenergy devices with geometry at the nanometer level, which provide new tools for bioelectrochemical studies. The use of individual 1D structure as electrodes for electrochemistry has opened a differentiated route toward electrochemical biomolecule studies. Silicon nanowires (SiNWs), as one dimensional structure, play more and more important role in nanoelectronics and optical devices with its unique attractive property. Moreover, with surface engineering treatment, the electrochemical properties of SiNWs at the Fermi level can be tuned with the interesting change from metallic to a nonmetallic character of the transport. Herein the surface engineering of SiNWs with enhancement of optical property addresses. Results show that due to the effect of surface engineering, the structure of SiNWs changes with higher efficient and stable photoluminescence without any luminescence decay, which definitely approve the significantly compelling effect of surface engineering of SiNWs.
\end{abstract}

Keywords: SiNWs, bioenergy, surface engineering, HF, nitridation
Volume 2 Issue 5 - 2018

\author{
Kelvii Wei GUO \\ Department of Mechanical and Biomedical Engineering, City \\ University of Hong Kong, China
}

\begin{abstract}
Correspondence: Kelvii Wei GUO, Department of Mechanical and Biomedical Engineering, City University of Hong Kong, 83 Tat Chee Avenue, Kowloon Tong, Kowloon, Hong Kong, China, Email kelvinguo@yahoo.com
\end{abstract}

Received: September 28, 2018 | Published: October 15, 2018

\section{Introduction}

Nanotechnology is the science and technology of precisely controlling the structure of matter at the nanometer scale. It shows exquisite new tools to engineer with novel materials and devices at the nanoscale. The materials and devices are generally considered to be in nano regime if the critical size is less than $100 \mathrm{~nm}$. As a unit of the length, a nanometer is one billionth of a meter, and about 10000 times narrower than a human hair. ${ }^{1}$ To date, it is well known that nanotechnology is now becoming the hottest research field which involves diverse fields such as physics, chemistry, biology, medicine, and optics. Nanotechnology has never been an independent discipline since its birth. On the basis of the unique physical, chemical or biological properties of nanostructures, nanotechnology creates the attractive materials with the interesting structures applied in various statuses..$^{2-4}$ Nanomaterials include clusters, thin films, wire structures and bulk nanostructured materials. Within the size range of nanometer, the optical, thermodynamic, mechanical, electronic, magnetic and chemical properties of nanomaterial are sufficiently different from individual atoms, molecules and bulk materials. The development of nanomaterials as well as the relevant understanding of its highlighted properties in recent years has enabled nanomaterials successful application in energy, electronics, medicine, chemical and biological sensors, etc. ${ }^{5-8}$ Due to the lack of the traditional energy resources, the most significant tendencies in the energy today is to minimize both economic inputs and environmental impacts for the long-run eco-friendly extremely goal. New promising energy have been recently developed with a progress in a material science, namely with an introduction of a wide range of nanomaterials. ${ }^{9-12}$

\section{Silicon nanowires (SiNWs)}

Since one-dimensional silicon nanowires(SiNWs) were synthesized by Lieber et al., ${ }^{13} \mathrm{SiNWs}$ have attracted much attention of research due to their potential significant application ranging from electronics and photonics to the life sciences and healthcare. Traditionally, surface modifications of materials means using chemical/physical methods to obtain or enhance certain surface properties without changing the bulk attributes. SiNWs are expected to have the potential application in bioenergy, especially in the optical areas. Photoluminescence (PL) is a unique property offered by SiNWs and has attracted more research in fabricating photoelectronics. However, because of the current development of an oxide layer covered on the surface of SiNWs, the degradation and the relatively low efficiency of the PL hinder its application further in energy field. Due to the absorption and reflection layer on the surface of $\mathrm{Si}$ with nanostructures, the increasing silicon oxide thickness with the exposure time to air causes degradation of PL intensity. ${ }^{14-17}$ In addition, the oxidization of Si with nanostructures inducts a blue shift of the PL peak due to the chemical instability of the surface. ${ }^{18,19}$ Therefore, a surface passivation must be urgently solved.

\section{Silicon nanowires (SiNWs) passivation}

Although a hydrogenation is easily available and can prevent certain degradation, it is not sufficient for application considerations. Hence, a better surface engineering must be employed to prevent the degradation. SiNWs have been successfully coated with the silicon carbide ( $\mathrm{SiC}$ ) using an ion beam deposition technique with methane $\left(\mathrm{CH}_{4}\right)$ as ion sources, which shows efficient and stable PL without the observable degradation. Various important gas-phase species for surface saturations have been explored theoretically. ${ }^{20-22}$ Among the existing passivation methods, HF and nitridation show the better performance than that of carbide. During the surface treatment, part of the surface oxide layer of SiNWs could be removed and the surface composition may be changed. Meanwhile, for the nitridation, there will be a nitride layer formed due to the reaction between the oxide and nitrogen species and the silicon nitride is air inert to the exposure and can prevent SiNWs from further oxidation.

\section{Silicon nanowires (SiNWs) HF treatment}

The silicon oxide layer of SiNWs serves as a protective layer rendering SiNWs relatively inert. The inertness of SiNWs is unfavorable for SiNWs applications. Thus, it is significant to 
improve the surface property of SiNWs to remove the oxide layer by HF etching. Figure 1 shows SEM image of SiNWs before surface engineering and HF-engineered SiNWs displays in Fig. 2. Results illustrate that upon etching with a dilute $(5 \%)$ aqueous HF solution, new absorption bands attributable to Si-Hx (2000-2200 and $\sim 900 \mathrm{~cm}-$ 1) and CHx (2800-3000 cm-1 due to organic impurities) are shown in the spectrum, while the $\mathrm{Si}-\mathrm{O}$ absorption bands virtually disappear. Moreover, hydrogen desorption due to the trihydrides occurs at $\sim 550$ $\mathrm{K}$ and that due to the dihydrides occurs at $\sim 650 \mathrm{~K}$. At or above 750 $\mathrm{K}$, all silicon hydride species begin to desorb from the surfaces of SiNWs. At about $850 \mathrm{~K}$, SiNWs surfaces are free of silicon hydride species. In addition, hydrogen-passivated surfaces of SiNWs show good stability in air (under ambient conditions) but relatively poor stability in water. ${ }^{23-26}$ The difference in the stabilities of hydrogenpassavited SiNWs in air versus in water may be associated with the hydroxide ion (even very low concentration in neutral solutions) in water because hydroxide ions can attack the silicon surface. The morphology of before surface engineering (Figure 1) changes considerably after etching with HF (Figure 2) and the diameter of the etched SiNWs reduces due to the removal of the outer oxide layer by HF engineering. Some Si nanoparticles form and absorp on the surface of SiNWs. Attributed to the sonication, the length of some SiNWs is shorted. These nanoparticles also increase the surface-tovolume ratio of after HF-engineered SiNWs.

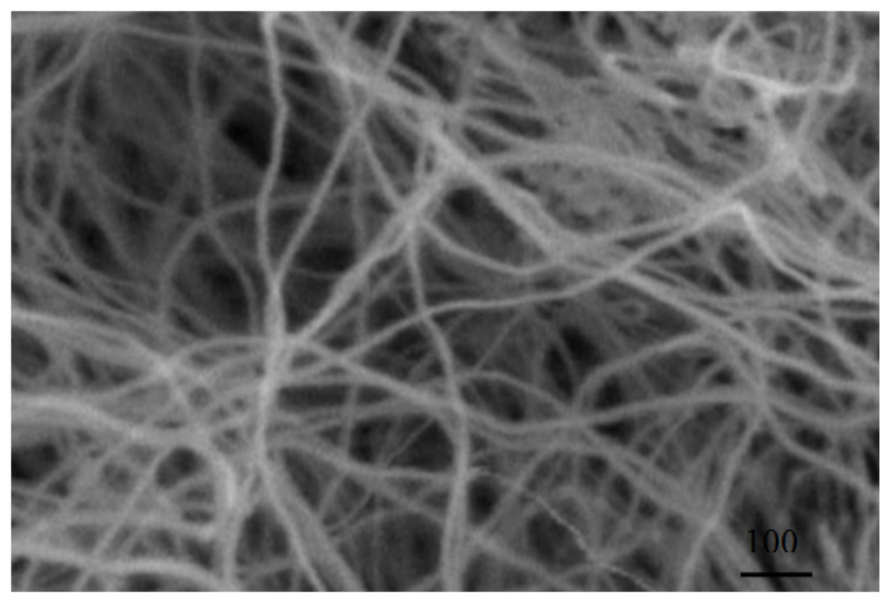

Figure I SiNWs before surface engineering.

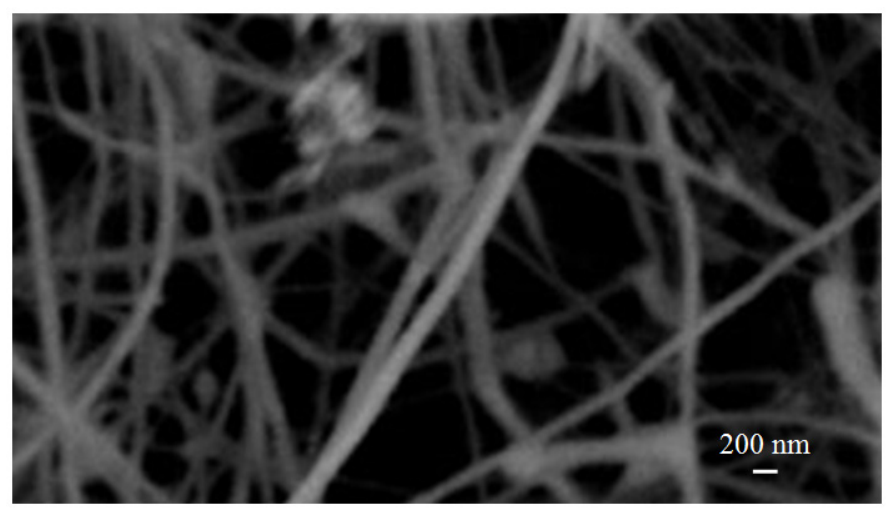

Figure 2 HF-engineered SiNWs.

Despite HF as a weak acid, it is quite active, allowing it to etch away the oxide layers in the reactions between HF and silicon/ silicon dioxide. In spite of the extraordinarily strong $\mathrm{Si}-\mathrm{F}$ bond, the highly polarized Si-F can be kinetically extremely reactive under ionic conditions, resulted in giving rise to H-terminated rather than F-terminated silicon surfaces. Although silicon generally forms the covalent bonds (with ionic character), it usually undergoes the ionic reactions, and/or participates in the bimolecular reactions, in which bond forming and bond breaking occurred simultaneously.

SiNWs treat with HF to remove the outer oxide layer of SiNWs, which decreases the electron transfer between the enzyme and the base electrode. The surfaces of the HF-etched, oxide-free SiNWs are passivated by hydrogen and exhibit moderately high reactivity. Results show that the amperometric response of the HF-etched SiNWs modified biosensor is greater than that of the as-grown SiNWs biosensor, which are consistent with the improved conductivity of SiNWs due to oxide removal by HF. HF-etched SiNWs provide both a suitable biocompatibility microenvironment for the entrapment of glucose oxidase as well as a mediator for the electron transfer between the enzyme and the electrode.

\section{Silicon nanowires (SiNWs) nitridation treatment}

Results of the nitride engineered SiNWs are shown in Figure 3 \& Figure 4, which indicate the morphologies of the nitride engineered SiNWs are smooth and uniform in diameter similar to the original SiNWs with the long, smooth and curved characteristic just shown as Figure 1. At higher temperature, the surface engineering effect leads to more efficient reaction on the surface, breaking SiNWs into thin and short pieces with the lower resolution under SEM observation. Results shown in Figure 3 \& Figure 4 are evidently more distinctive with better resolution compared with those shown in Figure 1, indicating that samples used in Figure 3 \& Figure 4 have better conductivity due to the reduction of the insolating silicon dioxide layer. It shows that at a temperature as high as $800^{\circ} \mathrm{C}$, SiNWs are completely broken into the amorphous silicon oxide nanoparticles. Although the reaction temperature is much lower than the melting temperature of silicon, but the reactant gas of nitride and oxygen cause the nanowire more ease to change and thus to form amorphous nanoparticles. Photoluminescence (PL) spectra of untreated SiNWs, treated at $600^{\circ} \mathrm{C}$, treated at $650^{\circ} \mathrm{C}$, and treated at $700^{\circ} \mathrm{C}$ show that with substrate temperature variation, PL peak, position and area vary simultaneously. SiNWs treated at different temperatures all display the stronger PL peaks in different wavelength regions than that of the untreated SiNWs. PL spectra have two to three peaks corresponding to silicon core (at higher wavenumber) and silicon oxide (at lower wavenumber). In addition, the spectrum shifts from the low wavenumber to the high wavenumber (red shift from 580 to $860 \mathrm{~nm}$ ) as the treatment temperature increases. It indicates that the domain of PL modifies (the silicon core reduces) at higher substrate temperatures and the red shift is due to the changes of the composition. Moreover, because of the removal of oxide layer and formation of silicon nitride, PL intensity enhances obviously at various temperatures.

FTIR spectra results show that there is not the absorption band related to silicon oxide for the untreated silicon nanowires because of the tightly packed surface atoms, which limit the mostly observed Si-O stretching mode to show up. With the increment of surface engineering temperature, Si-N stretching, N-H bending, Si-O stretching and $\mathrm{Si}-\mathrm{O}$ rocking modes show in the spectra. Both the Si-N stretching and $\mathrm{N}-\mathrm{H}$ bending modes indicate the nitridation of the surface layer silicon occurs by the surface treatment. The absorption peaks of $\mathrm{N}-\mathrm{H}$ bending mode are due to the NHy remained on the surface of SiNWs. The strong absorption at $1070 \mathrm{~cm}-1$ of Si-O stretching mode on the engineering surface indicates the effect of NHy ion leads the surface of SiNWs to be rough, and make the Si-O bonding free for stretching. 
Also, Si-O stretching enhances at the higher surface engineering temperature.

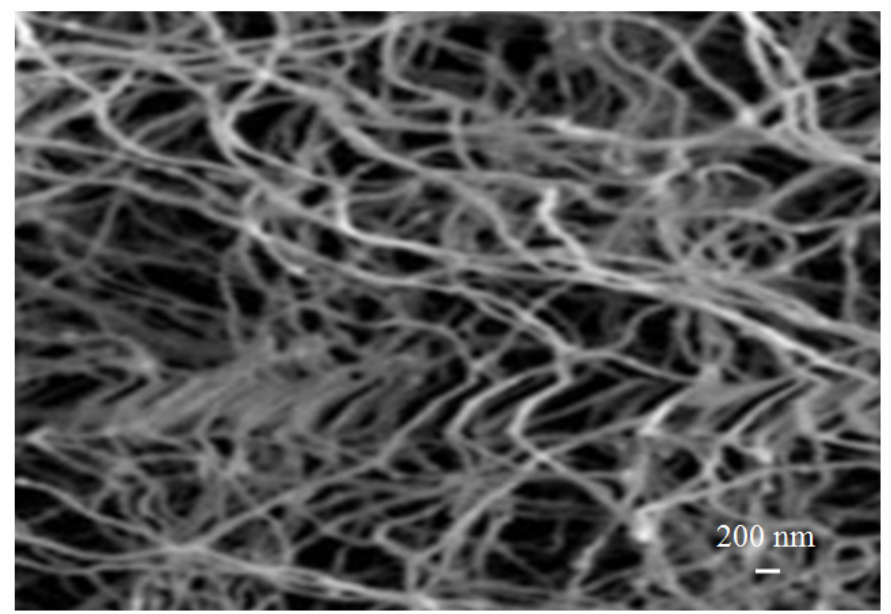

Figure 3 Engineering SiNWs at $600^{\circ} \mathrm{C}$.

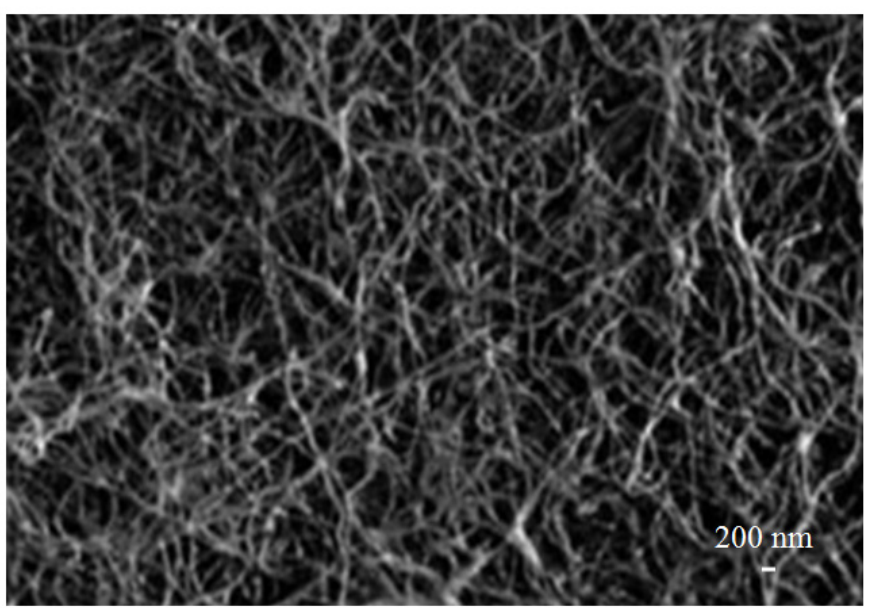

Figure 4 Engineering SiNWs at $650^{\circ} \mathrm{C}$.

The above-mentioned results elucidate that PL enhancement relates to the composition change of SiNWs. Before the SiNWs treated using $\mathrm{NH} 3$, the basic composition of SiNW includes a silicon core with a $\mathrm{Si}-\mathrm{SiO}$ interfacial layer containing silicon suboxide and a silicon dioxde layer at the surface. The silicon suboxide forms light barrier due to its small bandgap. After the surface nitride engineering, besides the surface nitride modification, the suboxide layer reduces with $\mathrm{N}$ addition into the interfacial layer. Consequently, SiWNs have better light emission behavior. It indicates that with $\mathrm{N}$ addition into the interfacial layer, the composition of silicon oxide changes with the SiWNs window opening. Therefore, silicon nanowires (SiNWs) with the various surface modifications (such as HF etching and nitridation) as the supporting matrixes are high sensitive to the glucose detection and the glucose oxidase (GOx) can be effectively adsorbed onto the treated SiNWs. When the decoration of SiNWs with hybrid materials (Pd, Ni and gold) is adopted, SiNWs can be made as the electrochemical methanol sensor with the definitely high sensitivity owing to SiNWs' high aspect ratio and materials compatibility with silicon substrate as electrode.

\section{Nanostructures for bioenergy}

In the field of biofuels energy, techniques related to nanotechnology can be taken as modification in feedstocks, higher efficient catalysts, etc. To date, enzymes have been utilized to catalyze biodiesel productions from oils and fats and to hydrolyze biomass to produce biofuels (for instance, ethanol and biogas) on a large scale. ${ }^{27-30} \mathrm{As}$ a captivating characteristics of nanostructures, they can be taken to replace or immobilize the enzymes with more efficient catalysis. ${ }^{31}$ Currently, biofuels have definitely attained attractive spot light and been manufactured in different countries along with an increasing production. Among them, for instance, bioethanol has the world production of around 25 millions of gallons per year. Biodiesel, another important biofuel, is usually produced by a chemically catalyzed transesterification reaction between low chain length alcohols (mainly methanol) and oils and fats from animals or plants. Up ot now, one of the most crucial potential materials for biofuel production is the vegetal biomass, mainly owing lignocellulosic compounds that are the most abundant renewable carbon source in the world. ${ }^{32}$ It is well known that nanotechnology is extremely beneficial to bioenergy production by changing the features of feed materials. Various kinds of nanomaterials, such as silicon nanowires, carbon nanotubes, magnetic and metal oxide nanoparticles and others are advantageous to become an essential part of sustainable bioenergy production. ${ }^{33,34}$ It is worthy noted that owing to nanostructures unique physical, chemical and electrical properties which differ from the bulk material, nanostructures can obviously improve the bioenergy production effectively.

\section{Applied in biogas production}

Biogas is produced from anaerobic digestion of organic wastes such as plant, agriculture and animal and human wastes. For enhancing the activity of methanogenic bacteria, which acts as a catalyst to the production of bioenergy, nanostructures are always adopted with the captivating results, such as the enhancement of the methane gas production. ${ }^{35,36}$ Moreover, the catalyst attached on nanostructures is more active and efficient, for instance, the enhancement of the activity of disintegration, the improvement of the yield of methane and biogas production. $^{37}$

\section{Applied in biofuel production}

It is noted that the first generation biofuel is produced from different food feedstocks such as starch from corn, sucrose from sugarcane, animal fats and plant oils. The second generation biofuel is used non-food feedstock (such as, wood wastes, agricultural residues, etc.). Although, second-generation biofuels have distinct advantages, they have some drawbacks such as high cost of production and infrastructure as well as technological problems. ${ }^{38}$ Owing to the properties of nanostructures, the abovementioned issues can be overcome because of its high surface to the volume ratio, quantum properties and immobilizing property. Biodiesel is a mixture of esters, which are commonly produced by transesterification of vegetable oils or animal fats with short chain alcohols (methanol or ethanol) that agree with specific standards to be used as fuel in diesel engines. ${ }^{39-42}$ Nanostructures with significant catalytic activities can be successfully used for the production of biodiesel from transesterification of glyceryl trioleate and distinctively increase in the biodiesel yield. ${ }^{43,44}$ Moreover, if nanostructures hybrid with other nanotechniques, such as magnetic nanocatalysts, they can be easily removed, recovered and reused, with the favoring challenge of the economic viability of the process with the traditional methods ${ }^{45,46}$ Results show that the catalysts with hybrid nanostructures possess considerable potential toward hydrolysis, transesterification, and esterification of soybean oil and fatty acids. ${ }^{47}$ 
Bioethanol (ethyl alcohol) is generally produced by carbon sources of sugarcane juice, grains, and others. These materials are mainly composed by cellulose and hemicellulose, polymeric structures of carbohydrates, and lignin. For the use of sugars present in cellulose and hemicellulose fractions, some pretreatments are required to break down the recalcitrance of biomass, disrupting polymeric fraction in fermentable monomers. ${ }^{48,49}$ Usually, after an initial pretreatment, cellulosic fraction of these materials is enzymatically hydrolyzed. By this method, monomeric glucose can be produced in milder conditions of process (lower temperature, without requirement of pressure), compared to chemical process, as well as the non-formation of undesirable fermentation inhibitor compounds..$^{50}$ Generally, the use of cellulases for the hydrolysis of lignocellulosic biomass is responsible for about $18 \%$ of total costs involved in the process of the bioethanol production. ${ }^{51,52}$ Therefore, the development of advanced strategies, which could provide the recovery and recycling of enzymes, can reduce production cost. Nanostructures have the ability to offer immobilization of various enzymes such as cellulases and hemicellulases, in the bioethanol production. It is promising to provide an easy way to recovery of enzyme with hybrid nanostructures that allow enzyme recovery and reuse for several cycles. The immobilization of enzyme on nanostructures is often achieved by covalent binding or physical adsorption. But, covalent binding method is more suitable because it can reduce protein desorption due to formation of covalent bonds between enzyme and nanostructures. Moreover, the performance of immobilized enzyme could be effectively controlled by tailoring nanostructures. As a result, the immobilized enzyme can be stored with longer storage which is potential to enhance in its thermostability property compared to free enzymes ${ }^{53}$ Also, enzyme immobilized on nanostructures by adsorption methods is successfully taken for the hydrolysis of lignocellulosic materials in the bioethanol production. Other beneficial contribution of nanostructures utilities, such as enhancement catalytic activity for production of ethanol, high efficacy of immobilized enzymes in hydrolysis of cellulose into glucose, increased yield of glucose, efficient simultaneous saccharification and fermentation, have been also achieved for recent years. ${ }^{54,55}$

\section{Silicon nanowires/nanostructures potential for bioenergy}

It is noted that the electrode materials with the nano functionalization and surface engineering techniques are usually taken to improve or enhance the direct electron transfer process, where for biofuel cells GOx is a definitely important enzyme, which suffers the electron transfer from its active center onto the electrode in the bioelectrocatalytic process. ${ }^{56}$ Owing to the unique optical and electrical properties of silicon nanowires (SiNWs) they are playing more and more significant role in the field of bioenergy. Generally, it will gain better efficiency in the electrochemical bioethanol sensors when the technology related to nano and micro is coupled with the fabricated systems. During SiNWs synthesis, its morphology, size and dope can be precisely controlled and the process of SiNWs fabrication is definitely high reproducible. Consequently, the fabricated sensor with $\mathrm{Pd}-\mathrm{Ni} / \mathrm{SiNW}$ shows higher sensitive to the methanol because of the high aspect ratio of the nanowires and the good compatibility with Si substrate as the electrode. ${ }^{57}$ Without SiNWs, the designed systems or devices for bioenergy deplete the current because of the poor electric contact between metal electrodes and silicon nanowires. As a result, the surface engineered SiNWs will definitely have the improved/enhanced good respond ability to the glucose sensitivity. Research shows that SiNWs are taken for the glucose detection with high sensitivity as the supporting matrixes successfully. ${ }^{58-60}$ After various treatments with $\mathrm{HF}$ etched and carboxylic acid $(\mathrm{COOH})$ functionalized, GOx can be efficiently adsorbed onto the surface of SiNWs. It indicates that the COOH-functionalized SiNWs amperometric biosensor has gained the superior detection sensitivity with the limit at $0.01 \mathrm{mM}$ glucose (the ratio of the signal to noise is 3 ).

\section{Conclusion}

With the surface engineering, silicon nanowires (SiWNs) have better surface properties potential for bioenergy. For SiNWs treatment with HF, the outer oxide layer of SiNWs removes to decrease the electron transfer between the enzyme and the base electrode, and the amperometric response of the HF-etched SiNWs is greater than that of the as-grown SiNWs, which indicates that SiNWs-modified electrode could respond to glucose sensitively. For SiNWs nitridation treatment, as photoluminescence (PL) enhancement with the composition of silicon oxide change and SiNWs possess higher efficient and stable photoluminescence without any luminescence decay than that of untreated SiNWs.

\section{Acknowledgements}

None.

\section{Conflict of interest}

Authors declare there is no conflict of interest in publishing the article.

\section{References}

1. Soriano ML, Zougagh M, Valcárcel M, et al. Analytical Nanoscience and Nanotechnology: Where we are and where we are heading. Talanta. 2018;177:104-121.

2. Oke AE, Aigbavboa CO, Semenya K. Energy Savings and Sustainable Construction: Examining the Advantages of Nanotechnology. Energy Procedia. 2017;142:3839-3843.

3. Contreras JE, Rodriguez EA, Taha Tijerina J. Nanotechnology applications for electrical transformers-A review. Electric Power Systems Research. 2017;143:573-584.

4. Hussein AK. Applications of nanotechnology to improve the performance of solar collectors-Recent advances and overview. Renewable and Sustainable Energy Reviews. 2016;62:767-792.

5. Lee IJ, Paik UY, Park JG. Solar cell implemented with silicon nanowires on pyramid-texture silicon surface. Solar Energy. 2013; 91: 256-262.

6. Srivastava SK, Singh P, Yameen M, et al. Antireflective ultra-fast nanoscale texturing for efficient multi-crystalline silicon solar cells. Solar Energy. 2015;115:656-666.

7. Basu PK, Khanna A, Hameiri Z. The effect of front pyramid heights on the efficiency of homogeneously textured inline-diffused screenprinted monocrystalline silicon wafer solar cells. Renewable Energy. 2015;78:590-598.

8. Altinoluk SH, Ciftpinar HE, Demircioglu O, et al. Light trapping by micro and nano-hole texturing of single crystalline silicon solar cells. Energy Procedia. 2016;92:291-296.

9. Kashyap D, Yadav RS, Gohil S, et al. Fabrication of vertically aligned copper nanotubes as a novel electrode for enzymatic biofuel cells. Electrochimica Acta. 2015;167:213-218.

10. Slaughter G, Kulkarni T. Fabrication of palladium nanowire array electrode for biofuel cell application. Microelectronic Engineering. 2016;149:92-96.

11. Adesina O, Anzai IA, Avalos JL, et al. Embracing biological solutions to the sustainable energy challenge. Chem. 2017;2:20-51. 
12. Cherfouh H, Fellahi O, Hadjersi $\mathrm{T}$, et al. CuInS2/SiNWs/S composite material for application as potential photoelectrode for photoelectrochemical hydrogen generation. International Journal of Hydrogen Energy. 2018;43:3431-3440.

13. Morales AM, Lieber CM. A laser ablation method for the synthesis of crystalline semiconductor nanowires. Science. 1998; 279(5348):208211.

14. Hidouri T, Saidi H, Amri C, et al. Effect of wavelengths and excitation density on the optical properties of P3HT: SiNWs bulk heterojunction for photovoltaic applications. Superlattices and Microstructures. 2016;97:409-416.

15. Amri C, Ouertani R, Hamdi A, et al. Effect of porous layer engineered with acid vapor etching on optical properties of solid silicon nanowire arrays. Materials \& Design. 2016;111:394-404.

16. Tingzon P, Lopez L, Oliver $\mathrm{N}$, et al. Terahertz emission and photoluminescence of silicon nanowires electrolessly etched on the surface of silicon (100), (110), and (111) substrates for photovoltaic cell applications. Photonics and Nanostructures-Fundamentals and Applications. 2017;24:1-6.

17. Saidi H, Walid A, Bouazizi A, et al. Effects of silicon nanowires (SiNWs) contents on the optical and dielectric properties of poly(3hexylthiophene):SiNWs nanocomposites. Journal of Physics and Chemistry of Solids. 2017;107:1-6.

18. Lajvardi M, Eshghi H, Izadifard M, et al. Effects of silver and gold catalytic activities on the structural and optical properties of silicon nanowires. Physica E: Low-dimensional Systems and Nanostructures. 2016;75:136-143.

19. Moumni B, Jaballah AB. Correlation between oxidant concentrations, morphological aspects and etching kinetics of silicon nanowires during silver-assist electroless etching. Applied Surface Science. 2017;425:1-7.

20. Prakash J, Venugopalan R, Tripathi BM, et al. Chemistry of one dimensional silicon carbide materials: Principle, production, application and future prospects. Progress in Solid State Chemistry. 2015;43(3):98122 .

21. Share K, Westover A, Li MY, et al. Surface engineering of nanomaterials for improved energy storage - A review. Chemical Engineering Science. 2016;154:3-19.

22. Soam A, Kavle P, Kumbhar A, et al. Performance enhancement of microsupercapacitor by coating of graphene on silicon nanowires at room temperature. Current Applied Physics. 2017;17(2):314-320.

23. Chandrasekaran S, Nann T, Voelcker NH. Nanostructured silicon photoelectrodes for solar water electrolysis. Nano Energy. 2015;17:308322 .

24. Aouida S, Benabderrahmane Zaghouani R, Bachtouli N, et al. Hydrogen passivation of silicon nanowire structures. Applied Surface Science. 2016;370:49-52.

25. Rahman T, Bonilla RS, Nawabjan A, et al. Passivation of all-angle black surfaces for silicon solar cells. Solar Energy Materials and Solar Cells. 2017;160:444-453.

26. Belhousse S, Tighilt FZ, Sam S, et al. Functionalization of silicon nanowires by conductive and non-conductive polymers. Applied Surface Science. 2017;421:134-141.

27. Minteer SD, Liaw BY, Cooney MJ. Enzyme-based biofuel cells. Current Opinion in Biotechnology. 2007;18(3):228-234.

28. Sokic Lazic D, de Andrade AR, Minteer SD. Utilization of enzyme cascades for complete oxidation of lactate in an enzymatic biofuel cell. Electrochimica Acta. 2011;56(28):10772-10775.

29. Azadi P, Malina R, Barrett SRH, et al. The evolution of the biofuel science. Renewable and Sustainable Energy Reviews. 2017; 76:14791484 .
30. Paramjeet S, Manasa P, Korrapati N. Biofuels: Production of fungalmediated ligninolytic enzymes and the modes of bioprocesses utilizing agro-based residues. Biocatalysis and Agricultural Biotechnology. 2018;14:57-71.

31. Kumar A, Sharma S, Pandey LM, et al. Nanoengineered material based biosensing electrodes for enzymatic biofuel cells applications. Materials Science for Energy Technologies. 2018;1(1):38-48.

32. Joshi G, Pandey JK, Rana S, et al. Challenges and opportunities for the application of biofuel. Renewable and Sustainable Energy Reviews. 2017;79:850-866.

33. Serrano E, Rus G, García-Martínez J. Nanotechnology for sustainable energy. Renewable and Sustainable Energy Reviews. 2009;13(9):23732384 .

34. Hussein AK. Applications of nanotechnology in renewable energies-A comprehensive overview and understanding. Renewable and Sustainable Energy Reviews. 2015;42: 460-476.

35. Swain PK. Utilisation of Agriculture Waste Products for Production of Bio-Fuels: A Novel Study. Materials Today: Proceedings. 2017;4(11):11959-11967.

36. Stephen JL, Periyasamy B. Innovative developments in biofuels production from organic waste materials: A review. Fuel. 2018; 214:623633.

37. Sikarwar VS, Zhao M, Fennell PS, et al. Progress in biofuel production from gasification. Progress in Energy and Combustion Science. 2017;61:189-248.

38. Rodionova MV, Poudyal RS, Tiwari I, et al. Biofuel production: Challenges and opportunities. International Journal of Hydrogen Energy. 2017;42(12):8450-8461.

39. Mardhiah $\mathrm{HH}$, Ong $\mathrm{HC}$, Masjuki $\mathrm{HH}$, et al. A review on latest developments and future prospects of heterogeneous catalyst in biodiesel production from non-edible oils. Renewable and Sustainable Energy Reviews. 2017;67:1225-1236.

40. Balamurugan T, Arun A, Sathishkumar GB. Biodiesel derived from corn oil-A fuel substitute for diesel. Renewable and Sustainable Energy Reviews. 2018;94:772-778.

41. Gebremariam SN, Marchetti JM. Economics of biodiesel production: Review. Energy Conversion and Management. 2018;168: 74-84.

42. Suresh M, Jawahar CP, Richard A. A review on biodiesel production, combustion, performance, and emission characteristics of non-edible oils in variable compression ratio diesel engine using biodiesel and its blends. Renewable and Sustainable Energy Reviews. 2018; 92:38-49.

43. Banković Ilić IB, Miladinović MR, Stamenković OS, et al. Application of nano CaO-based catalysts in biodiesel synthesis. Renewable and Sustainable Energy Reviews. 2017;72:746-760.

44. Muthusamy S, Nallathambi SS, Ramasamy RK, et al. Effects of nanoparticles blended biodiesel on single cylinder CI Engine. Materials Today: Proceedings. 2018;5(2):6831-6838.

45. El Nahas AM, Salaheldin TA, Zaki T, et al. Functionalized cellulosemagnetite nanocomposite catalysts for efficient biodiesel production. Chemical Engineering Journal. 2017;322:167-180.

46. Souza DR, Vats T, Chattree A, et al. Graphene supported magnetically separable solid acid catalyst for the single step conversion of waste cooking oil to biodiesel. Renewable Energy. 2018;126:1064-1073.

47. Loman AA, Ju LK. Soybean carbohydrate as fermentation feedstock for production of biofuels and value-added chemicals. Process Biochemistry. 2016;51(8):1046-1057.

48. Srivastava N, Srivastava M, Mishra PK, et al. Applications of fungal cellulases in biofuel production: Advances and limitations. Renewable and Sustainable Energy Reviews. 2018;82(3):2379-2386. 
49. Onumaegbu C, Mooney J, Alaswad A, et al. Pre-treatment methods for production of biofuel from microalgae biomass. Renewable and Sustainable Energy Reviews. 2018;93:16-26.

50. Kumari D, Singh R. Pretreatment of lignocellulosic wastes for biofuel production: A critical review. Renewable and Sustainable Energy Reviews. 2018;90:877-891.

51. Rasmussen M, Abdellaoui S, Minteer SD. Enzymatic biofuel cells: 30 years of critical advancements. Biosensors and Bioelectronics. 2016;76:91-102.

52. Cosnier S, Gross AJ, Goff AL, et al. Recent advances on enzymatic glucose/oxygen and hydrogen/oxygen biofuel cells: Achievements and limitations. Journal of Power Sources. 2016;325:252-263.

53. de Poulpiquet A, Ciaccafava A, Gadiou R, et al. Design of a H2/O2 biofuel cell based on thermostable enzymes. Electrochemistry Communications. 2014;42:72-74.

54. Neto SA, Almeida TS, Palma LM, et al. Hybrid nanocatalysts containing enzymes and metallic nanoparticles for ethanol/O2 biofuel cell. Journal of Power Sources. 2014;259:25-32.
55. Bhalla A, Bansal N, Kumar S, et al. Improved lignocellulose conversion to biofuels with thermophilic bacteria and thermostable enzymes. Bioresource Technology. 2013;128:751-759.

56. Adesina O, Anzai IA, Avalos JL, et al. Embracing biological solutions to the sustainable energy challenge. Chem. 2017;2(1):20-51.

57. Noy A, Artyukhin AB, Misra N. Bionanoelectronics with 1D materials. Materials Today. 2009;12(9):22-31.

58. Horng YY, Hsu YK, Ganguly A, et al. Direct-growth of polyaniline nanowires for enzyme-immobilization and glucose detection. Electrochemistry Communications. 2009;11:850-853.

59. Holzinger M, Goff AL, Cosnier S. Carbon nanotube/enzyme biofuel cells. Electrochimica Acta. 2012;82:179-190.

60. Yazdi AA, Angelo DL, Omer N, et al. Carbon nanotubemodification ofmicrobialfuelcellelectrodes. Biosensors and Bioelectronics. 2016;85:536-552. 